\title{
Downregulation of microRNA-196a inhibits human liver cancer cell proliferation and invasion by targeting FOXO1
}

\author{
LIU YANG $^{1}$, FEI PENG ${ }^{2}$, JIAN QIN ${ }^{3}$, HENGHUA ZHOU $^{4}$ and BING WANG ${ }^{3}$ \\ ${ }^{1}$ Department of Gastroenterology, Shanghai Ninth People's Hospital, Shanghai Jiaotong University, School of Medicine; \\ ${ }^{2}$ Department of Surgery, Jingan Branch of Huashan Hospital, Fudan University; \\ ${ }^{3}$ Department of Surgery, Shanghai Ninth People's Hospital, Shanghai Jiaotong University, School of Medicine; \\ ${ }^{4}$ Department of Pathology, Shanghai Ninth People's Hospital, School of Medicine, \\ Shanghai Jiaotong University, Shanghai, P.R. China
}

Received February 10, 2017; Accepted July 21, 2017

DOI: 10.3892/or.2017.5873

\begin{abstract}
Hepatocellular carcinoma (HCC) is the most common type of primary liver cancer in adults and effective therapy for human liver cancer remains a difficult clinical concern. Researchers have demonstrated that microRNAs play important roles in the tumorigenesis and tumor progression of human liver cancer; therefore, regulation of microRNAs may be a new strategy for HCC therapy. MicroRNA-196a (miR196a) has been reported to be overexpressed in many types of cancers. However, the regulatory effects of miR-196a in human liver cancer are not fully understood. In the present study, we found that miR-196a was overexpressed in human liver cancer cells compared to that observed in normal liver cells. MTT and colony formation assays indicated that downregulation of miR-196a inhibited liver cancer cell proliferation which was due to the induction of cell apoptosis. A mouse model demonstrated that downregulation of miR-196a also inhibited human liver cancer cell migration and invasion in vivo. Further study indicated that FOXO1 is a direct target of miR-196a, and inhibition of FOXO1 promoted human liver cancer cell growth. Taken together, the present study demonstrated that the expression of miR-196a in human liver cancer cells was upregulated; downregulation of miR-196a regulated human liver cancer cell biological functions which could benefit the clinical therapy of human liver cancer in the future.
\end{abstract}

\section{Introduction}

Hepatocellular carcinoma (HCC) is a common cancer worldwide (1). In China, the incidence of HCC accounts for more than $50 \%$ of all cancer cases (2). Only $5 \%$ of HCC patients

Correspondence to: Dr Bing Wang, 639 Zhizao Ju Road, Department of Surgery, Shanghai Ninth People's Hospital, Shanghai, P.R. China

E-mail: bingw_sh@yeah.net

Key words: miR-196a, human liver cancer cells, proliferation, FOXO1 survive more than five years (3), which is mainly attributed to late diagnosis. Patients who undergo surgical therapy usually have a high recurrence rate which may be another reason for the low survival rate. Therefore, a full understanding of the mechanisms underlying HCC tumorigenesis and tumor progression is needed for improving the diagnosis and clinical therapy of HCC. Recently accumulating studies have demonstrated that microRNAs, a type of non-coding small RNAs play important biological roles in the tumorigenesis and tumor progression of HCC, providing new insights into the therapy of HCC (4-7).

MicroRNAs (miRNAs) are a series of small non-coding RNAs, formed by $21-24$ nucleotides, and they regulated gene expression by targeting mRNAs, which may lead to mRNA degradation or translation suppression $(8,9)$. Recently, studies indicate that deregulation of miRNAs causes the regulation of cell proliferation, differentiation and apoptosis, and these biological functions are important in cancer development $(10,11)$. Several studies reported that miR-196a expression is upregulated in many human cancers including non-small cell lung cancer, gastric and breast cancer (12-15). miR-196a is transcribed from miR-196a-1 and miR-196a-2; miR-196a plays important biological roles in the development of human cancer (16-18). However, the biological role of miR-196a in human liver cancer is not well established. In the present study, we evaluated the expression of miR-196a in human liver cancer cells, investigated the biological roles of miR-196a and aimed to ascertain the mechanisms underlying the effects of miR-196a on human liver cancer cells.

\section{Materials and methods}

Cell lines and animals. All the liver cancer cell lines were purchased from the Type Culture Collection of the Chinese Academy of Sciences (Shanghai, China). Cells (Huh7, MHCC-97H, Hep3B, HepG2 and SMMC-7721) were cultured in Dulbecco's modified Eagle's medium (DMEM) which was supplemented with fetal bovine serum (FBS) (1:10), penicillin (1:100) and streptomycin (1:100) (all from Invitrogen, Carlsbad, CA,USA). Primary human hepatocytes (PHHs) and PHH medium were purchased from the Research Institute for Liver Diseases Co. Ltd. (Shanghai, China). BALB/c nude mice (4-5 weeks of 
age) (female) were purchased from SLAC Laboratory Animal Co., Ltd. (Shanghai, China). Mice were housed in an SPF animal facility for at least 7 days before the start of the present study. All animal studies were performed according to the requirements of the 'Guide for the Care and Use of Laboratory Animals' [National Research Council (US) Committee].

miR-196a quantification. The total RNA was extracted from the human liver cancer cells using TRIzol. cDNA was transcribed from the extracted total RNA using a TaqMan ${ }^{\circledR}$ MicroRNA Reverse Transcription kit (Applied Biosystems ${ }^{\mathrm{TM}}$ ). qPCR assay was performed for the quantification of miR-196a; the PCR assay was performed using TaqMan ${ }^{\circledR}$ Universal PCR Master Mix (Applied Biosystems ${ }^{\mathrm{TM}}$ ). The primers used were: miR-196a forward, CGTCAGAAGGAATGATGCACAG and reverse, ACCTGCGTAGGTAGTTTCATGT. RNA U6 was used as an internal control; the primers used for U6 were forward, CTCGCTTCGGCAGCACA and reverse, AACGCT TCACGAATTTGCGT. The miR-196a expression was normalized with U6. Real-time PCR was performed on a LightCycler 480 Real-Time PCR System (Roche Diagnostics, Basel, Switzerland) 40 cycles of $95^{\circ} \mathrm{C}$ for $10 \mathrm{sec}$, and $60^{\circ} \mathrm{C}$ for $30 \mathrm{sec})$.

Transfection assay. Human liver cancer cells were cultured for $24 \mathrm{~h}$ in DMEM which was supplemented with FBS. Cultured cells were transfected with the miR-196a or miR control inhibitor using Lipofectamine ${ }^{\circledR} 3000$ reagent (Invitrogen ${ }^{\mathrm{TM}}$ ). Both miR-196a inhibitor and miR control inhibitor were purchased from Exiqon, Inc. (Woburn, MA, USA). The final concentration of inhibitor used was $10 \mathrm{nM}$. For the FOXO1 knockdown assay, the present study was performed according to a previously described protocol (19). The siRNA was purchased from Santa Cruz Biotechnology, Inc. (Santa Cruz, CA, USA).

MTT and colony formation assays. For the MTT assay, human liver cancer cells were transfected with miR-196a or miR control inhibitor, and cultured (3,500 cells/well) in a 96-well plate. The cell proliferation was evaluated every $24 \mathrm{~h}$ using an MTT kit (cat. no. C0009, Beyotime Institute of Biotechnology, Nantong, China) following the manufacturer's protocol. For colony formation assay, human liver cancer cells were transfected with miR-196a or control inhibitor and seeded into a 6 -well plate (500 cells/well). Cells were cultured for another 2 weeks, and then the cells were fixed with methanol and stained with crystal violet; the numbers of colonies were counted. All the experiments were performed in triplicate.

Cell invasion assays. For the cell invasion assay, transfected cells were placed into the upper chamber of an insert coated with Matrigel (500,000 cells/well). Cell culture medium with FBS was added into the lower culture chamber. After a 12-h incubation, the cells remaining on the upper membrane (not migrated) were removed by washing with cold phosphatebuffered saline (PBS) twice; while the cells that had migrated were fixed and stained with crystal violet solution. The number of cells was counted using an inverted microscope. Each sample was counted in five random fields.
Cell apoptosis assay. Human liver cancer cells were transfected with miR-196a or miR control inhibitor, and cultured for $48 \mathrm{~h}$. Then, the cells were harvested and stained with FITC-Annexin V and propidium iodide. FACS assay was performed to evaluate cell apoptosis using flow cytometry. Data were analyzed using CellQuest software (both from BD Biosciences, San Jose, CA, USA). All the experiments were performed in triplicate.

Experimental metastasis assay in vivo. To investigate the effect of miR-196a on human liver cancer cell metastasis in vivo, a mouse model was used. Mock and transfected HepG2 cells were injected into 4 - to 5 -week-old nude mice via tail vein $(4,000,000$ cells/mouse; $0.2 \mathrm{ml})$. Four weeks later, the mice were sacrificed and the mouse liver tissues were harvested. Then, the number of metastatic tumor nodules were counted under a dissecting microscope.

Dual-luciferase assay. To construct the FOXO1 luciferase report vector, the wild-type (WT) or mutant (Mut) FOXO1 gene was cloned downstream of the luciferase gene in the pLUC luciferase vector. Then, the reporter vectors were cotransfected with the miR-196a mimic or control mimic into HepG2 cells using Lipofectamine ${ }^{\circledR} 3000$ reagent (Invitrogen). Cells were cultured for another $48 \mathrm{~h}$, and then harvested and lysed for the luciferase assay using a luciferase assay kit. Relative luciferase activity was normalized with the Renilla luciferase activity. All the experiments were performed in triplicate.

Real-time PCR. Human liver cancer cells were transfected with miR-196a inhibitor or control inhibitor and cultured. The total RNA was extracted from the human liver cancer cells using TRIzol (Invitrogen). cDNA was transcribed from the extracted total RNA using Reverse Transcriptase MMLV (Takara, Shiga, Japan). PCR reactions were performed as followed: $95^{\circ} \mathrm{C}$ for $5 \mathrm{~min}$, followed by 45 cycles of $15 \mathrm{sec}$ at $95^{\circ} \mathrm{C}, 30 \mathrm{sec}$ at $60^{\circ} \mathrm{C}$ and $15 \mathrm{sec}$ at $72^{\circ} \mathrm{C}$. The primers used for FOXO1 were purchased from Bio-Rad (Hercules, CA, USA). The primers used were: GAPDH forward, GGTGATGCTGGTGCTGAGTA and reverse, ACTGTGGTCATGAGCCCTTC. The relative expression of FOXO1 was determined using the $2^{-\Delta \Delta \mathrm{Ct}}$ method. All the experiments were performed in triplicate.

Western blotting. Human liver cancer cells were transfected with miR-196a or control inhibitor and cultured for $48 \mathrm{~h}$, and then cells were harvested. Protein was extracted from the cells and separated using SDS-polyacrylamide gel. Then, the separated protein was transferred to an NC membrane. The NC membrane was blocked with blocking buffer for $120 \mathrm{~min}$ and then submitted to primary antibody incubation at RT for another $120 \mathrm{~min}$. The NC membrane was washed with TBST three times and then the NC membrane was incubated with the secondary antibody for $120 \mathrm{~min}$. The NC membrane was washed with Tris-buffered saline with Tween-20 (TBST) for three times again and the target protein was examined using ECL chemiluminescence and exposure to X-ray film. All the experiments were performed in triplicate. 


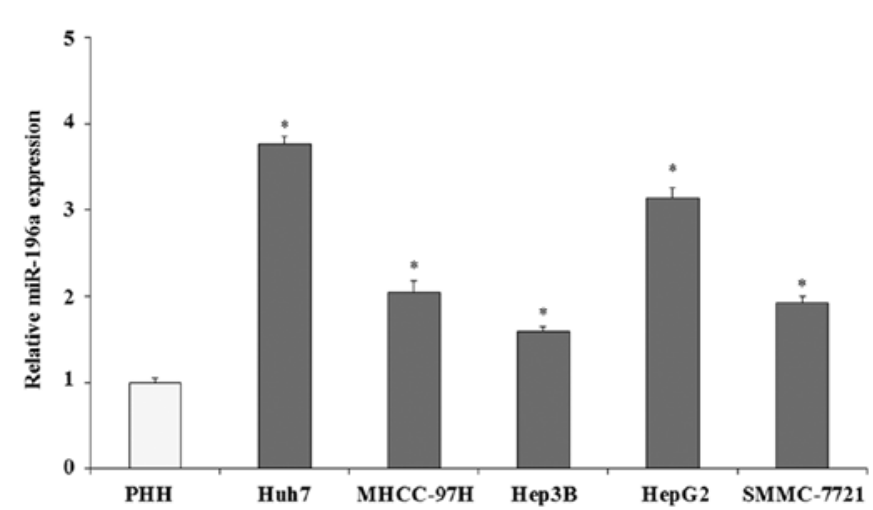

Figure 1. Expression of miR-196a in human liver cancer cell lines. The expression of miR-196a was detected by qPCR assay. The data are expressed as the mean $\pm \mathrm{SD} ;{ }^{*} \mathrm{p}<0.05$.

Statistical analysis. Student's t-test (two-tailed) and one-way ANOVA were performed to analyze statistical differences using SPSS 16.0 software (SPSS, Inc., Chicago, IL, USA). For in vitro study, the data are expressed as mean $\pm \mathrm{SD}$; for in vivo study the data are expressed as mean \pm SEM. $p<0.05$ indicates a statistically significant.

\section{Results}

miR-196a is overexpressed in human liver cancer cells. The expression of miR-196a in human liver cancer cells was evaluated by qRT-PCR assay. The expression of miR-196a was normalized with U6 (U6 was used as an internal control). The qRT-PCR results demonstrated that the expression of miR-196a in five liver cancer cell lines (Huh7, MHCC-97H, Hep3B, HepG2 and SMMC-7721) was significantly increased compared with that in the PHHs (Fig. 1 ; p<0.05), particularly in HepG2 (3.14-fold; $\mathrm{p}<0.05$ ) and Huh7 cells (3.76-fold; $\mathrm{p}<0.05)$. These findings indicate that miR-196a is overexpressed in human liver cancer cells. We hypothesized that miR-196a plays important roles in the progression and development of human liver cancer. In the present study, we selected the two cell lines, HepG2 and Huh7, with high miR-196a expression to investigate the biological roles of miR-196a in human liver cancer.

miR-196a manipulation in human liver cancer cells. In order to selectively inhibit the expression of miR-196a, miR-196a inhibitor transfection assay was performed. HepG2 or Huh7 cells were cultured and transfected with miR-196a inhibitor or miR inhibitor control and cultured for another $48 \mathrm{~h}$. The expression of miR-196a was then examined by qRT-PCR assay. The results showed that after miR-196a inhibitor transfection the expression of miR-196a was significantly decreased to 0.21 -fold in the HepG2 cells and 0.23 -fold in the Huh7 cells, respectively, compared with the mock HepG2 or Huh7 cells (Fig. 2; p<0.05). In contrast, in the miR inhibitor control-transfected cells there were no significant changes in the expression of miR-196a (Fig. 2; p>0.05).

Effect of miR-196a on human liver cancer cell proliferation and invasion. To better understand the biological roles of miR-196a in the progression and development of human liver

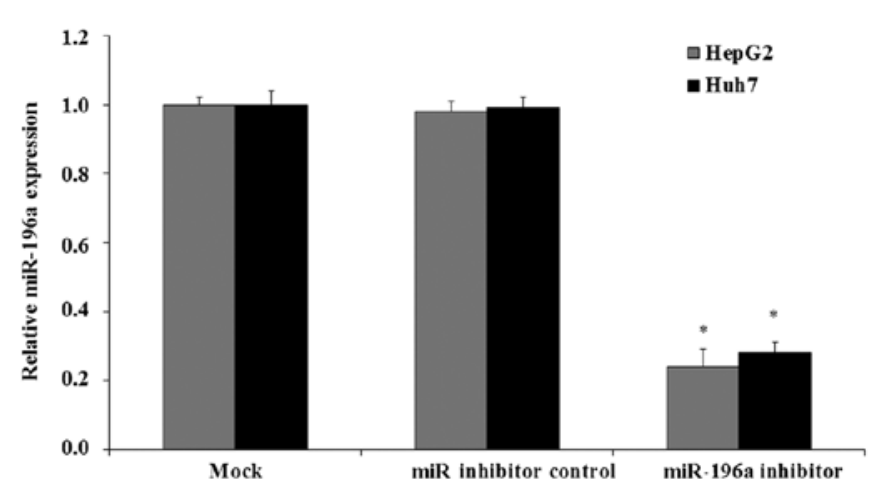

Figure 2. Expression of miR-196a in mock- and miR inhibitor-transfected human liver cancer cells. Expression of miR-196a was detected by qPCR assay. The data are expressed as the mean $\pm \mathrm{SD} ;{ }^{*} \mathrm{p}<0.05$.

cancer, we assessed the effect of miR-196a on liver cancer cell proliferation. Human liver cancer HepG2 and Huh7 cells were seeded into 96-well plates and the cell proliferation was evaluated by MTT assay every $24 \mathrm{~h}$. MTT results revealed that after miR-196a inhibitor transfection the cell proliferation was significantly inhibited when compared to that noted in the cells transfected with the miR inhibitor control and the mock group (Fig. 3A and B; p<0.05). Then, we performed a colonyformation assay to confirm the cell proliferation inhibition. As we expected the results demonstrated that downregulation of miR-196a significantly decreased the colony formation number both in the HepG2 and Huh7 cells (Fig. 3C; p<0.05). Further study indicated that downregulation of miR-196a significantly inhibited human liver cancer cell invasion in vitro (Fig. 3D; $\mathrm{p}<0.05)$.

Effect of miR-196a on human liver cancer cell apoptosis. In order to investigate whether the HepG2 and Huh7 cell growth inhibition was attributed to cell apoptosis, FACS assay was performed. HepG2 and Huh7 cells were transfected with miR196a or miR inhibitor control and cultured for $24 \mathrm{~h}$, and then the HepG2 and Huh7 cells were harvested and submitted to a FACS assay. Our results showed that the downregulation of miR-196a by inhibitor transfection increased the HepG2 and Huh7 cell apoptosis, while the miR inhibitor control transfection did not affect HepG2 and Huh7 cell apoptosis (Fig. 4; p<0.05). Therefore, downregulation of miR-196a induced human liver cancer cell apoptosis.

miR-196a promotes proliferation, migration and invasion of HepG2 cells in vivo. The previous results demonstrated that inhibition of miR-196a suppressed human liver cancer HepG2 and Huh7 cell growth in vitro. We hypothesized that downregulation of miR-196a would also inhibit liver cancer cell growth and migration in vivo. We confirmed the hypothesis using an in vivo mouse model. Mice were modeled by the injection of HepG2 cells (mock or transfected) via tail vein; each mouse was injected with $4 \times 10^{6}$ cells. Mice were maintained in an SPF animal room for six weeks, and then the mice were sacrificed and the mouse livers were harvested; the metastatic nodules were counted. The results showed that in the mock and the miR inhibitor control group there were a few large metastatic tumors (data not shown); however, large tumors 
A

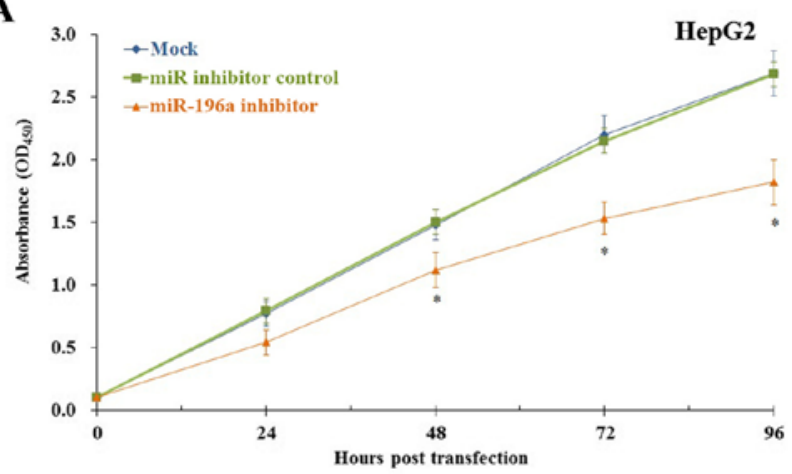

B

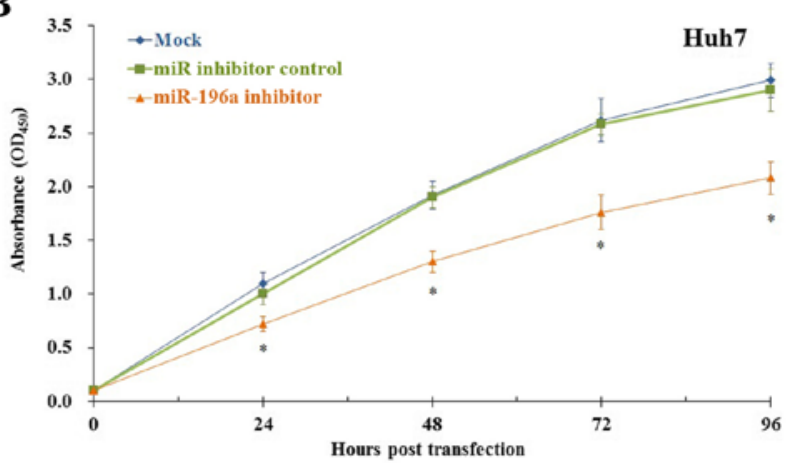

C

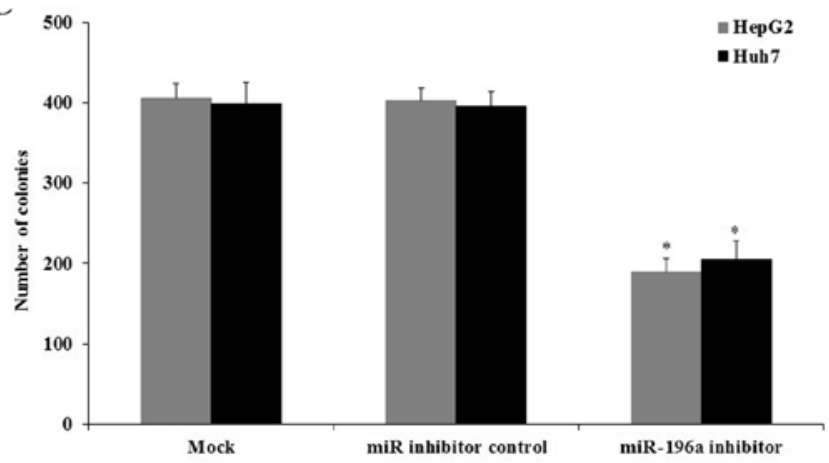

D

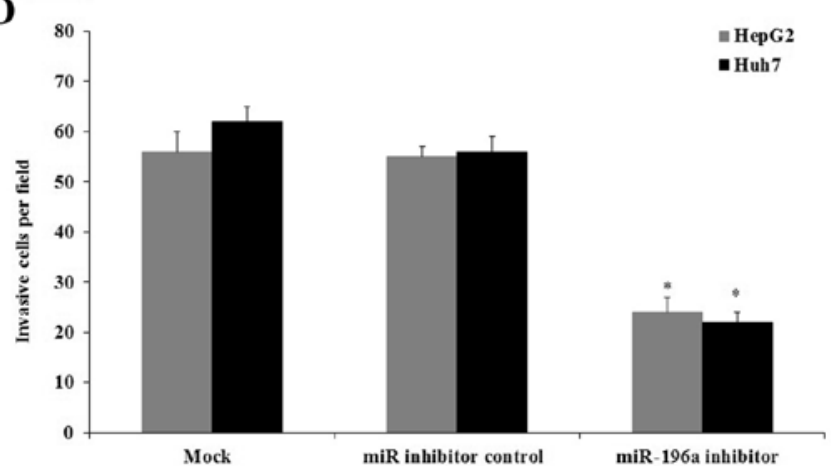

Figure 3. Effect of miR-196a on human liver cancer cell proliferation and invasion. Cell viability was measured by MTT assay. (A) Effect of miR-196a on HepG2 cell proliferation. (B) Effect of miR-196a on Huh7 cell proliferation. (C) Effect of miR-196a on liver cancer cell colony formation. (D) Effect of miR-196a on liver cancer cell invasion. The data are expressed as the mean $\pm \mathrm{SD} ;{ }^{*} \mathrm{p}<0.05$.

were not observed in the miR-196a inhibitor group. Tumor counting results showed that the number of metastatic nodules
A

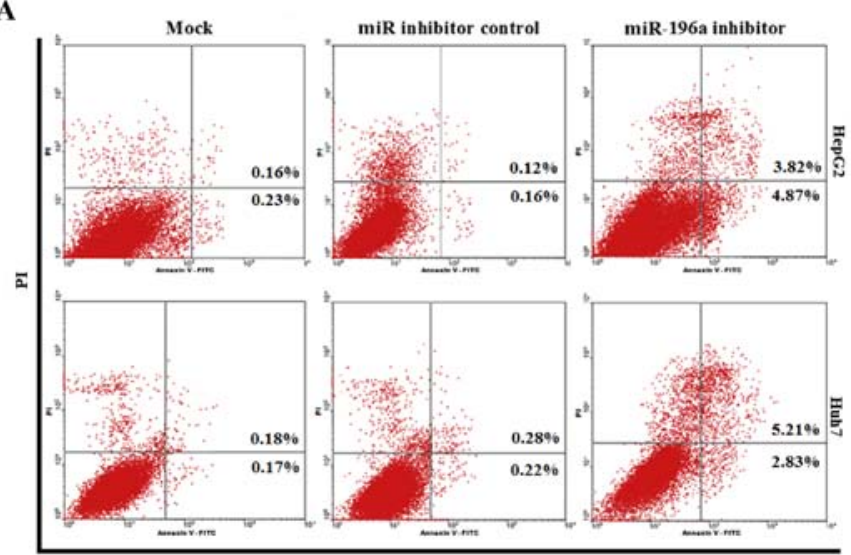

Annexin V - FTrC

B

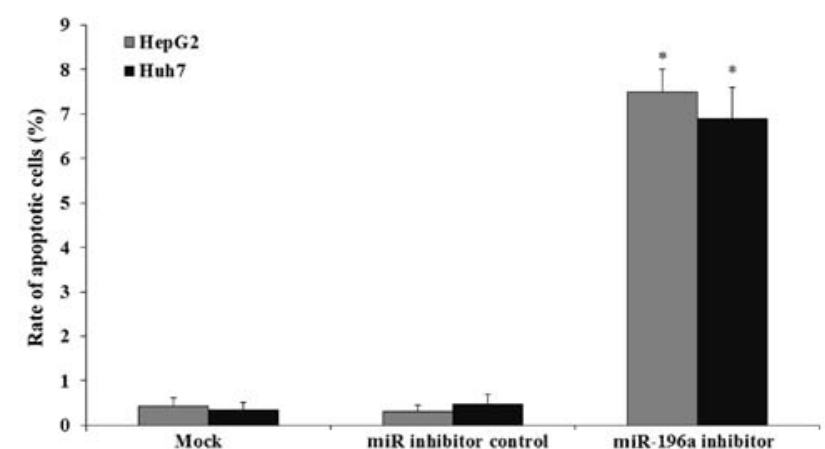

Figure 4. Downregulation of miR-196a induces human liver cancer cell apoptosis. (A) The percentage of apoptotic cells was determined by FACS (B) Histogram showing the percentage of apoptotic cells. Each bar in the histogram represents the mean $\pm \mathrm{SD} ;{ }^{*} \mathrm{p}<0.05$.

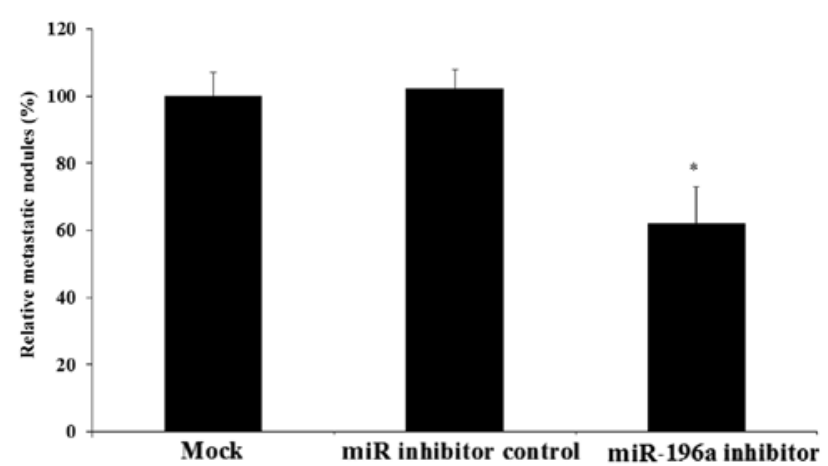

Figure 5. A metastatic mouse model was constructed by the injection of HepG2 cells (mock or transfected) via tail vein. After 6 weeks, the mice were sacrificed and the livers were harvested; the metastatic nodules were counted. The number of metastatic nodules was decreased significantly in the miR-196a inhibitor group compared with that in the miR inhibitor control and mock group $(* \mathrm{p}<0.05)$.

was decreased significantly in the miR-196a inhibitor group compared with that in the miR inhibitor control and mock group (Fig. 5; $\mathrm{p}<0.05$ ). Therefore, the present study indicated that the inhibition of miR-196a decreased the proliferation and migration of HepG2 cells in vivo.

FOXO1 is a direct target of miR-196a. To better understand the molecular mechanisms by which miR-196a regulates the 
A

CMV promoter Luciferase FOXO1 3' UTR_ Poly A

WT 3' UTR 5'-UAAACUUUUGUUUGUACUACCUG-3' miR-196a 3'-GGGUUGUUGUACUUUGAUGGAU-5' Mut 3'UTR 5'-UAAACUUUUGUUUGUUACGCGUG-3'

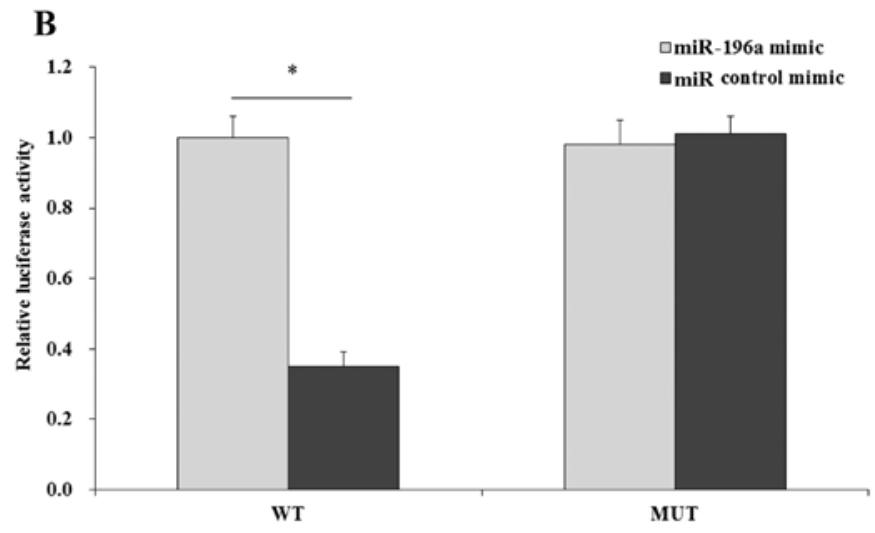

Figure 6. FOXO1 is a direct target of miR-196a. (A) The binding sites of miR-196a in the human FOXO1 gene. (B) Dual-Luciferase assay confirmed that FOXO1 is a direct target of miR-196a. The data are expressed as the mean $\pm \mathrm{SD} ;{ }^{*} \mathrm{p}<0.05$.

behavior of human liver cancer cells, we aimed to identify the direct target of miR-196a. FOXO1 was found to be a potential target of miR-196a by TargetScan program search (Fig. 6A). Next, we investigated whether FOXO1 is a direct target of miR-196a by dual-luciferase assay. The WT 3' untranslated region (3'UTR) of FOXO1 was directly fused downstream of the firefly luciferase gene, and the miR-196a mimic and various luciferase 3'UTR constructs (WT, Mut) were co-transfected into HepG2 cells. miR control mimic was used as the control. Cells were cultured and luciferase activity was determined. The results indicated that induction of miR-196a significantly decreased the luciferase activity of the pLuc-FOXO1 3'UTR reporter (WT), while we did not observe significant inhibition between the miR-196a mimic and miR control mimic in the Mut group (Fig. 6B), therefore FOXO1 was identified as a direct target of miR-196a.

Effect of FOXO1 on human liver cancer cells. To examine the expression of FOXO1 in human liver cancer cells, qRT-PCR assay was performed. The expression of FOXO1 was significantly decreased in the HepG2 and Huh7 cells compared to that noted in the PHHs ( $\mathrm{p}<0.05$; Fig. 7A). To selectively regulate the expression of FOXO1 in human liver cancer cells, RNAi knockdown assay was performed. As shown in Fig. 7B, qRT-PCR (a) and western blotting (b) results indicated that after the transfection of si-FOXO1, the expression of FOXO1 in the HepG2 cells was significantly decreased both at mRNA and protein level compared to the levels noted in the si-NC-treated HepG2 cells $(\mathrm{p}<0.05)$. As shown in Fig. 7C, MTT (a) and colony formation (b) assays demonstrated that the downregulation of FOXO1 promoted HepG2 cell proliferation $(\mathrm{p}<0.05)$. Cell invasion study indicated that downregulation of FOXO1 promoted HepG2 cell invasion ( $<<0.05$; Fig. 7C-c). Then, we performed FACS assay to evaluate the effect of FOXO1 on HepG2 cell apoptosis.

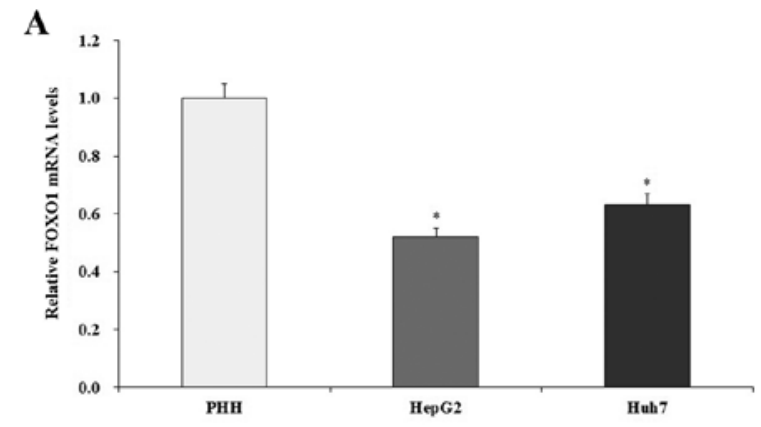

B a

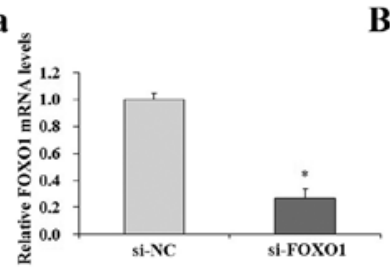

B b

$\mathrm{C}$ a

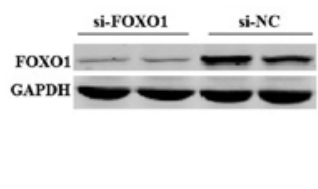

C b
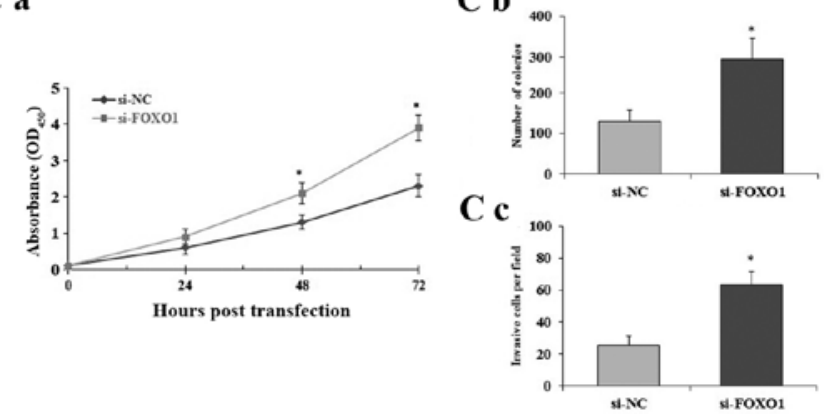

D

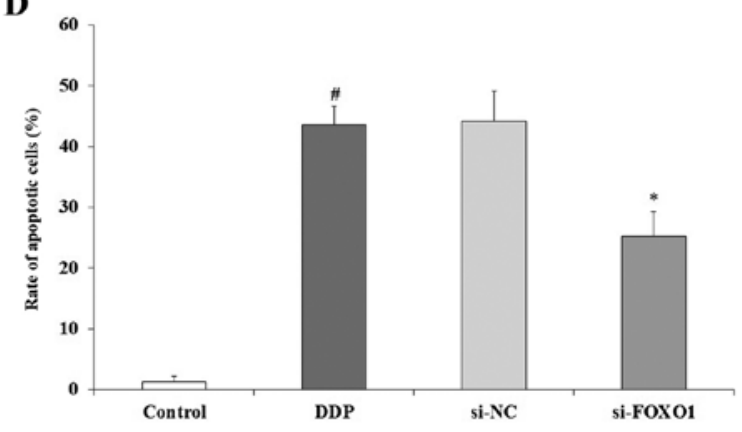

Figure 7. FOXO1 inhibits the cell growth and promotes the cell apoptosis of human liver cancer cells. (A) The expression of FOXO1 in human liver cancer cells was evaluated by qRT-PCR assay. (B) The expression of FOXO1 was decreased both at the mRNA (a) and protein (b) level by siRNA assay. (C) Suppression of FOXO1 increased human liver cancer cell proliferation (a), colony formation number (b) and cell invasion (c). (D) Suppression of FOXO1 inhibited the apoptosis of cells treated with cisplatin (DDP). The data are expressed as the mean $\pm \mathrm{SD} ;{ }^{*} \mathrm{p}<0.05$.

FACS results indicated that downregulation of FOXO1 decreased the apoptosis of HepG2 cells induced by cisplatin (DDP) ( $<<0.05$; Fig. 7D). All these data suggest that FOXO1 expression inhibits human liver cancer HepG2 cell growth and induces cell apoptosis.

miR-196a modulates liver cancer cell proliferation by suppressing FOXO1. As miR-196a was found to be upregulated in human liver cancer cells and FOXO1 is a direct target of miR-196a and regulates the growth of human liver cancer cells, we next aimed to determine whether miR-196a 
A

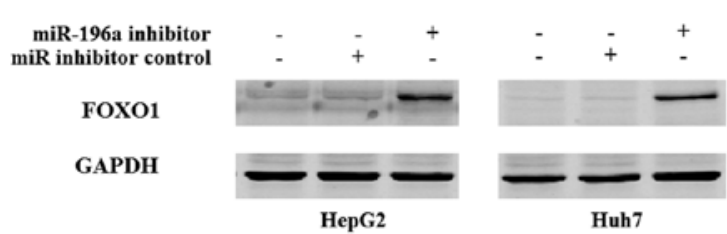

B

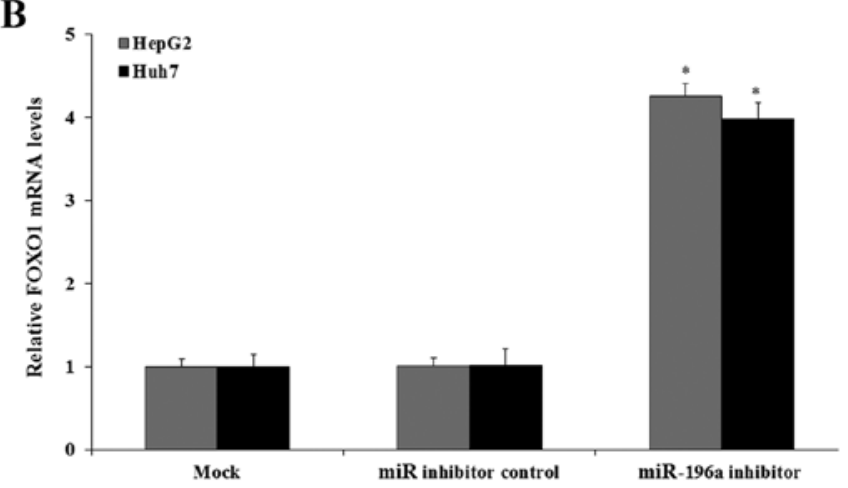

C

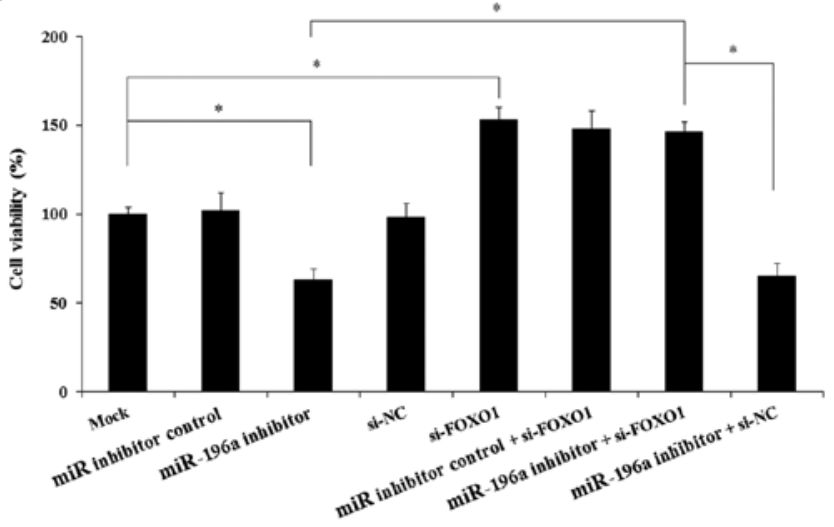

D

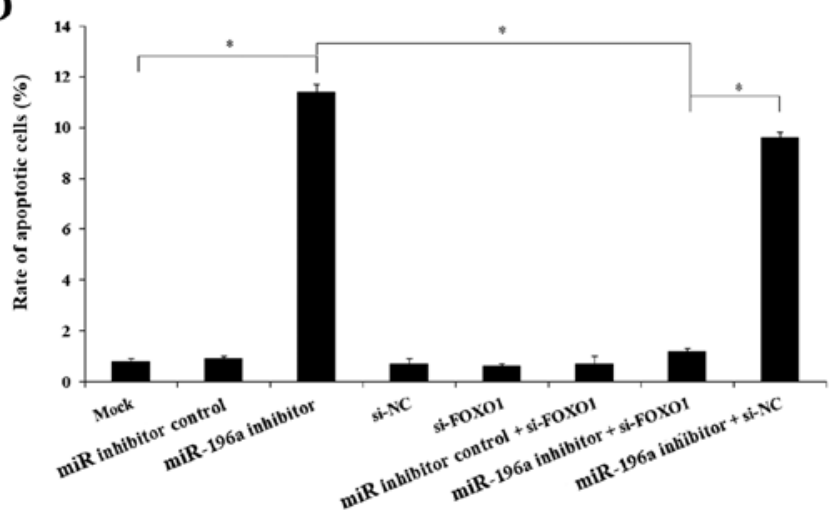

Figure 8. miR-196a regulates human liver cancer cell functions by targeting FOXO1. (A) Expression of FOXO1 protein in human liver cancer cells by western blot assay. (B) The expression of FOXO1 mRNA in human liver cancer cells following transfection by qPCR assay. (C) The cell viability of Huh7 cells was evaluated by MTT assay $72 \mathrm{~h}$ after transfection. (D) The cell apoptosis of Huh7 cells was evaluated by FACS assay $72 \mathrm{~h}$ after transfection. The data are expressed as the mean $\pm \mathrm{SD} ;{ }^{*} \mathrm{p}<0.05$

regulates human liver cancer cell growth by the regulation of FOXO1. miR-196a inhibitor and miR inhibitor control were transfected into HepG2 and Huh7 cells, and the expression of FOXO1 was examined. The results indicated that downregulation of miR-196a significantly increased the expression of FOXO1 both at the mRNA and protein levels compared with the miR inhibitor control and mock group $(\mathrm{p}<0.05$; Fig. $8 \mathrm{~A}$ and B). Further study indicated that following knockdown of the FOXO1 gene by si-FOXO1, suppression of miR-196a did not inhibit Huh7 cell proliferation (Fig. 8C) and also did not induce cell apoptosis (Fig. 8D) in the Huh7 cells. Therefore, miR-196a regulates the growth of human liver cancer cells by targeting FOXO1 which is a direct target of miR-196a.

\section{Discussion}

miRs are a series of small and non-coding RNAs. Recently studies have demonstrated that miRs play important roles in the tumorigenesis and tumor progression of HCC (4-7). Research also indicates that miRs regulate the expression of several genes and pathways (CDK, Ci/Kip, PI3K/AKT and mTOR) to regulate human liver cancer cell proliferation (20). Previous studies have shown that miR-196a is upregulated in breast, colon, gastric, pancreatic and cervical cancer, and miR-196a promotes cancer cell proliferation by the negative regulation of tumor-suppressor genes (FOXO1, p27, IкB $\alpha$, netrin 4 and ING5) (21-23). FOXO1 and p27Kip1 are direct targets of miR-196a in human cervical cancer (21). However the biological function of miR-196a in human liver cancers are still not well explored. In the present study, we found that the expression of miR-196a was upregulated in human liver cancer cells compared to that noted in normal human liver cells. Downregulation of miR-196a inhibited human liver cancer cell proliferation and invasion in vitro and in vivo which were attributed to the induction of cell apoptosis.

FOXO1 is an important member of the FOXO family. The FOXO1 gene regulates several biological functions of cancer cells including cancer cell proliferation, cancer cell differentiation, cancer cell apoptosis and angiogenesis (24). Tumorigenesis is closely connected with cell proliferation, cell cycle and cell apoptosis, while FOXO regulates cell proliferation and cell cycle by the regulation of related genes, such as: p53, p27Ki p1, cyclin B, cyclin D1/D2 and cyclin G2 (24-26). Previous research revealed the FOXO1 is weakly expressed in liver cancer tissue, which results in abnormal cell proliferation and cell apoptosis; while the induction of FOXO1 expression inhibited liver cancer cell proliferation and induced cell apoptosis by the regulation of p21, p27 and cyclin D1 (26). In the present study, we demonstrated that FOXO1 is a direct target of miR-196a. Inhibition of FOXO1 significantly promoted human liver cancer cell proliferation and downregulation of miR-196a induced the expression of FOXO1.

Taken together, the present study showed that miR-196a is overexpressed in human liver cancer cells, and the downregulation of miR-196a inhibited human liver cancer cell proliferation and induced human liver cancer cell apoptosis which was attributed to the regulation of FOXO1, a direct target of miR-196a. Therefore, the present study suggests a new vision and strategy for the therapy of liver cancer. However, the detailed mechanisms underlying the effects of miR-196a on liver cancer warrant further investigation. 


\section{References}

1. Parkin DM: Global cancer statistics in the year 2000. Lancet Oncol 2: 533-543, 2001.

2. El-Serag HB and Rudolph KL: Hepatocellular carcinoma: Epidemiology and molecularcarcinogenesis. Gastroenterology 132: 2557-2576, 2007.

3. Hao K, Luk JM, Lee NP, Mao M, Zhang C, Ferguson MD, Lamb J, Dai H, Ng IO, Sham PC, et al: Predicting prognosis in hepatocellular carcinoma after curative surgery with common clinicopathologic parameters. BMC Cancer 9: 389, 2009.

4. Drakaki A, Hatziapostolou M and Iliopoulos D: Therapeutically targeting microRNAs in liver cancer. Curr Pharm Des 19: 1180-1191, 2013.

5. He XX, Chang Y, Meng FY, Wang MY, Xie QH, Tang F, Li PY, Song YH and Lin JS: MicroRNA-375 targets AEG-1 in hepatocellular carcinoma and suppresses liver cancer cell growth in vitro and in vivo. Oncogene 31: 3357-3369, 2012.

6. Liang L, Wong CM, Ying Q, Fan DN, Huang S, Ding J, Yao J, Yan M, Li J, Yao M, et al: MicroRNA-125b suppressesed human liver cancer cell proliferation and metastasis by directly targeting oncogene LIN28B2. Hepatology 52: 1731-1740, 2010.

7. Huang $\mathrm{S}$ and He $\mathrm{X}$ : The role of microRNAs in liver cancer progression. Br J Cancer 104: 235-240, 2011.

8. Farazi TA, Spitzer JI, Morozov P and Tuschl T: miRNAs in human cancer. J Pathol 223: 102-115, 2011.

9. Bartel DP: MicroRNAs: Genomics, biogenesis, mechanism, and function. Cell 116: 281-297, 2004.

10. Chitwood DH and Timmermans MC: Small RNAs are on the move. Nature 467: 415-419, 2010.

11. Kosik KS: MicroRNAs and cellular phenotypy. Cell 143: 21-26, 2010.

12. Hui AB, Shi W, Boutros PC, Miller N, Pintilie M, Fyles T, McCready D, Wong D, Gerster K, Waldron L, et al: Robust global micro-RNA profiling with formalin-fixed paraffin-embedded breast cancer tissues. Lab Invest 89: 597-606, 2009.

13. Wang YX, Zhang XY, Zhang BF, Yang CQ, Chen XM and Gao HJ: Initial study of microRNA expression profiles of colonic cancer without lymph node metastasis. J Dig Dis 11: 50-54, 2010.

14. Sun M, Liu XH, Li JH, Yang JS, Zhang EB, Yin DD, Liu ZL, Zhou J, Ding Y, Li SQ, et al: MiR-196a is upregulated in gastric cancer and promotes cell proliferation by downregulating p27 (kip1). Mol Cancer Ther 11: 842-852, 2012.
15. Wang R, Wang ZX, Yang JS, Pan X, De W and Chen LB: MicroRNA-451 functions as a tumor suppressor in human non-small cell lung cancer by targeting ras-related protein 14 (RAB14). Oncogene 30: 2644-2658, 2011.

16. Braig S, Mueller DW, Rothhammer T and Bosserhoff AK: MicroRNA miR-196a is a central regulator of HOX-B7 and BMP4 expression in malignant melanoma. Cell Mol Life Sci 67: 3535-3548, 2010

17. Guan Y, Mizoguchi M, Yoshimoto K, Hata N, Shono T, Suzuki SO, Araki Y, Kuga D, Nakamizo A, Amano T, et al: MiRNA-196 is upregulated in glioblastoma but not in anaplastic astrocytoma and has prognostic significance. Clin Cancer Res 16: 4289-4297, 2010

18. Schimanski CC, Frerichs K, Rahman F, Berger M, Lang H, Galle PR, Moehler M and Gockel I: High miR-196a levels promote the oncogenic phenotype of colorectal cancer cells. World J Gastroenterol 15: 2089-2096, 2009.

19. Du Y, Zhu M, Zhou X, Huang Z, Zhu J, Xu J, Cheng G, Shu Y, Liu P, Zhu W, et al: MiR-20a enhances cisplatin resistance of human gastric cancer cell line by targeting NFKBIB. Tumour Biol 20: 1-9, 2015

20. Huang F, Tang J, Zhuang X, Zhuang Y, Cheng W, Chen W, Yao H and Zhang S: MiR-196a promotes pancreatic cancer progression by targeting nuclear factor kappa-B-inhibitor alpha. PLoS One 9: e87897, 2014

21. Hou T, Ou J, Zhao X, Huang X, Huang Y and Zhang Y: MicroRNA-196a promotes cervical cancer proliferation through the regulation of FOXO1 and p2 $7^{\mathrm{Kipl}}$. Br J Cancer 110: 1260-1268, 2014.

22. Zhang J, Zheng F, Yu G, Yin Y and Lu Q: miR-196a targets netrin 4 and regulates cell proliferation and migration of cervical cancer cells. Biochem Biophys Res Commun 440: 582-588, 2013.

23. Liu M, Du Y, Gao J, Liu J, Kong X, Gong Y, Li Z, Wu H and Chen H: Aberrant expression miR-196a is associated with abnormal apoptosis, invasion, and proliferation of pancreatic cancer cells. Pancreas 42: 1169-1181, 2013.

24. Zhao Y, Wang Y and Zhu WG: Applications of post-translational modifications of FoxO family proteins in biological functions. J Mol Cell Biol 3: 276-282, 2011

25. Zhang Y, Gan B, Liu D and Paik JH: FoxO family members in cancer. Cancer Biol Ther 12: 253-259, 2011.

26. Carbajo-Pescador S, Mauriz JL, García-Palomo A and GonzálezGallego J: FoxO proteins: Regulation and molecular targets in liver cancer. Curr Med Chem 21: 1231-1246, 2014. 